\title{
Design and Performance Analysis of B15 Engine Intake System
}

\author{
Shirong Wen, Baolei Wei, Huabei Tong \\ School of Mechanical Engineering and Automation, Chongqing Institute of Mechanical and Electrical Engineering, \\ Chongqing, China
}

\section{ABSTRACT}

In recent years, with the development of the economy, the automobile industry has also undergone ever-changing changes. At present, the design of the engine in addition to the car to achieve the dynamic, economic indicators but also need to meet their emissions and noise requirements, it is necessary for the engine into the exhaust system for in-depth study. The intake system mainly comprises two parts of the pipeline and the intake manifold before the intake manifold, in which the pipeline in front of the manifold acts to reduce the intake noise. How to design the components of the intake system reasonably and analyze its comprehensive performance is the most important topic in the project.

Firstly, this article introduces the principle of variable valve timing and variable intake manifold technology, establishes B15 engine model in GT-Power software and uses the test data to complete the calibration of the model so that the error is not more than $5 \%$ for subsequent simulation calculations. In order to design the silencer element of the intake system, the sensitivity of the parameters of the Helmholtz resonator, 1/4 wavelength tube and air filter is calculated and analyzed, and the influence of the parameter change on the muffler performance is summarized. The design flow and method of the intake muffler element are described in detail, and the concrete design scheme is put forward. The noise of the muffler element is optimized according to the problem that the noise of the individual order is higher than the target value. After the optimization of the intake system air intake noise improved significantly, the pressure loss is no higher than $2.5 \mathrm{kPa}$, in line with the target value requirements, the optimization effect is good.

Secondly, in order to analyze the performance of the intake manifold, three methods are proposed: the zerodimensional or one-dimensional model is used to simplify the processing, and the CFD software is used to calculate the steady-state or isolated transients and the coupling analysis using one-dimensional and three-dimensional software. By comparing the advantages and disadvantages of the three methods, it is found that the coupling of software can obtain realtime boundary condition in the calculation process and have high accuracy. This paper focuses on the key issues that need to be solved by coupling with GT-Power and STAR-CCM + software and completes the calculation of the model.

Finally, in order to evaluate the advantages and disadvantages of the intake muffler element better, the maximum noise of the intake system is calculated by using the model of the vehicle acceleration in the GT-Power software, and the maximum noise of the intake system is $61.8 \mathrm{~dB}(\mathrm{~A})$ which satisfies the target requirement for the individual contribution rate through noise.

Keywords: B15 engine design, Exhaust and intake system, GT-Power, noise and emission control

\section{Received: $18^{\text {th }}$ Jan. $2019 \quad$ Accepted: $6^{\text {th }}$ Apr. $2019 \quad$ Online: $20^{\text {th }}$ Apr. 2019}

\section{Introduction}

\subsection{Research background}

In recent years, various countries have put forward higher and higher requirements for automobile noise, making the study of automobile noise is admitted no delay. To reduce the noise of car, first to determine and figure out the source and the factor of the noise. The car is an integrated noise source that contains many different types of noise, such as engine noise, wind noise, tire noise, road noise and resonance noise. The correlation test shows that the noise generated by the engine is the largest noise, which belongs to the structural noise, and the change of the engine speed is manifested in

Copyright (C) 2019 Wen et al. This is an Open Access article distributed under the terms of the Creative Commons Attribution-NonCommercial 4.0 International License (http://creativecommons.org/licenses/by-nc/4.0/), permitting all non-commercial use, distribution, and reproduction in any medium, provided the original work is properly cited. 
different forms, mainly through the front leaf board, the firewall, the hood and the exhaust pipe. Hence, the way to reduce engine noise effectively has become the central link in reducing vehicle noise technology. Combustion noise, aerodynamic noise, and mechanical noise are engine noise, and aerodynamic noise includes intake noise, exhaust noise, and fan noise. Statistical analysis of the test results shows that in the main noise source of the engine, the intake and exhaust noise accounts for a large proportion. In most of the cars, the engine exhaust noise is the most important source of noise, it is more obvious than the intake noise [1]. In order to effectively eliminate the exhaust noise, in the exhaust system to install the muffler has become a universal means, this technology is also relatively mature. For small high-speed machines and large turbochargers, intake air noise is second only to the main noise source of exhaust noise and sometimes higher than the engine's ontology noise.

As the state of the vehicle noise requirements gradually becomes high, mere consideration of the elimination of exhaust noise has been unable to meet the requirements of national laws and regulations, it is necessary to study how to eliminate the intake noise effectively. The function of the engine intake system is as much as possible and as even as possible to provide flammable mixtures or pure air to each qihong. General intake system mainly includes the intake manifold before the pipeline (such as the intake manifold, air filter and other silencers, etc.) and intake manifold. In some carburetor and throttle body gasoline jet engine, the general will be equipped with preheating the air intake device. In order to obtain the effect of enhanced intake, some of the engine intake systems are also equipped with a resonator for the gasoline jet engine, the intake system is also equipped with metering air device. Air fiter is an important role in filtering out air impurities and dust, for the gas to provide clean air. In addition, since the air cleaner structure is an inflated cavity, similar to the expansion type muffler structure, but also has the role of reducing the intake noise, so the engine intake system generally avoid installing a separate air intake muffler. The noise control can be studied from two aspects: First, the control of the noise source, the second is to take external noise reduction measures, such as the design of silencer components. The control of intake air noise is mainly through the design of the matching intake system muffler components to achieve, which is currently the most effective and the most useful methods. By installation space constraints, intake muffler components are generally integrated with the air filter [2]. On some engines where the air filter cannot meet the noise reduction requirements, the corresponding Hermes muffler or $1 / 4$ wavelength tube can also be installed. The way to design an intake muffler element under the premise of satisfying the engine power and economy is a very important process. In addition to the manifold before the intake manifold, the engine's intake system also includes the intake manifold itself. The effect of intake manifolds on reducing air intake noise is small and basically it can be neglected. However, its physical structure is still very important. Since the air necessary for the normal operation of the engine flows through the intake manifold through the intake manifold and finally into the cylinder, the influence of the manifold structure on the flow field during the flow of the fluid is clearly manifested. A good structure can minimize friction, eliminate flow resistance so that the flow of fluid as smooth as possible. Therefore, the analysis of the manifold flow field is necessary.

\subsection{Research status}

\subsubsection{Overview of foreign research}

The study of the engine intake system started relatively early in the country, and there was a simple calculation and test for the car noise before the 1960s. For example, Hosomi. Mikiya et al. summarized the development of the engine exhaust muffler, the development of simulation technology, structural analysis was the first to be used in the automotive field, using finite element components to calculate the mode and response. In the 1970s, the finite element method was used to study the internal structure of the muffler. The three-dimensional finite element analysis was carried out in the 1980s, and a simple calculation of the transmission loss of the muffler was completed. The realization of these techniques shows that the 3D finite element analysis method has a good effect on the structure of the muffler. Later, numerical simulation technology has been widely used, the engine simulation technology is also gradually mature, such as Bhattacharya. Paritosh and others through the numerical simulation software to complete the two rain four-stroke diesel engine performance analysis and noise research. Computational fluid dynamics (CFD) technology was born in the 1970s in the United States and is widely used in a variety of fluid flow and heat transfer problems. For four decades, CFD has made rapid development 
in the aspects of lightning flow model, grid technology and parallel computing, and brought revolutionary changes to the industry. In the automotive industry, CFD and other computer aids are used as product development tools to significantly reduce the number of prototype tests. In 1994, Thomas Morel and Leon A. LaPointe proposed the concept of 'parallel simulation and experimentation', complementing simulation and experimentation, implementing an integrated solution to establish a numerical model, and using the basic data collected by the experiment to correct the model and perfect, and get the data that cannot collected from the experiment, in the computer parallel simulation and test to complete the engine design optimization and development. In the actual simulation calculation, in order to avoid the use of three-dimensional software alone that is not high accurate, the researchers proposed a one-dimensional and three-dimensional software called the computational fluid dynamics method to analyze the flow characteristics of the muffler, through the calculation results predict the pressure loss and acoustic performance of the engine in the internal and external exhaust system under the actual operating conditions, so as to realize the pre-optimization [3].

\subsubsection{Overview of domestic research}

China's research on the intake system started late in 1979, after the promulgation of motor vehicle standards began to implement the experimental development as the focus of the study. With the link between China and the world, the automobile industry is also facing great challenges, the urgent need for independent research and development of new products, in which the control of noise has become one of the topics cannot be avoided. As China's awareness on the noise pollution is not enough, making the current research on the noise reduction technology and developed countries have a certain gap, the ability to start relatively low. With the increasing requirements of noise regulations, only the exhaust system muffler has been unable to meet the noise reduction requirements, the urgent need to develop the intake system research. In recent years, many domestic car developers aware of this problem, many experts and scholars have done lots of work. Shanyong Mao and others through the comparison of the engine in different conditions of the sound pressure cloud, indicating that the high-speed engine intake air and intake noise on the machine noise has a significant impact. Qiwang Fan and others use Matlab to write the program to simulate the influence of the structural parameters of the Helmholtz resonance muffler on the muffler performance. It is found that the volume and the cross-sectional area of the resonant cavity have important influence on the resonant frequency and the muffler. The use of GT-Power software to analyze the insertion loss of the intake system and the center frequency design expert system of the intake air muffler component are taken into account. The parameters of the transmission loss are taken into account, and the air filter, Helmholtz resonance Cavity and $1 / 4$ wavelength tube, this system has a high practical value for the design of the acoustic components of the intake system. Yan Jin, Zhiyong Hao By comparing the spectral characteristics of the noise outside the driving, the application experiment and the boundary element method The frequency of the corresponding characteristics of the original structure on the basis of the Helmholtz resonator, has become a good solution to the problem of intake noise, and already achieved the purpose of vehicle noise reduction. Xiaoyan Zhang et al. through the establishment of the engine model, optimized the intake and exhaust system, the variable intake manifold system for a complete modeling, improved model simulation accuracy. Ling Yin et al. analyzes the influence of two kinds of intake manifolds on the dynamic performance of the whole machine by analyzing the design scheme of different intake manifolds by using one-dimensional and threedimensional software. At the same time, it solves the problem that the three-real-time change of boundary conditions, resulting in the results of the problem is not high accurate, so that the simulation results are closer to the test values of these research results for the future design and optimization of the intake system has a very important significance.

\section{Design of B15 Engine Intake Muffler Components}

\subsection{Overview of the intake system}

The engine's air intake system is an assembly of air or gas into the air and red parts, which functions as the supplier to supply combustible mixture or clean, dry air and as much as possible. The main components of the system air filter, piping, design and installation are directly impact on the engine's power and economy. 


\subsubsection{Air intake system structure and working principle}

The air intake system includes an air inlet system and an air intake manifold system, wherein the former mainly refers to the intake manifold, besides which includes an air cleaner, an intake silencer, an intake pipe and a flexible connection pipe, Figure 1 shows [4].

Figure 1. Schematic diagram of the intake system

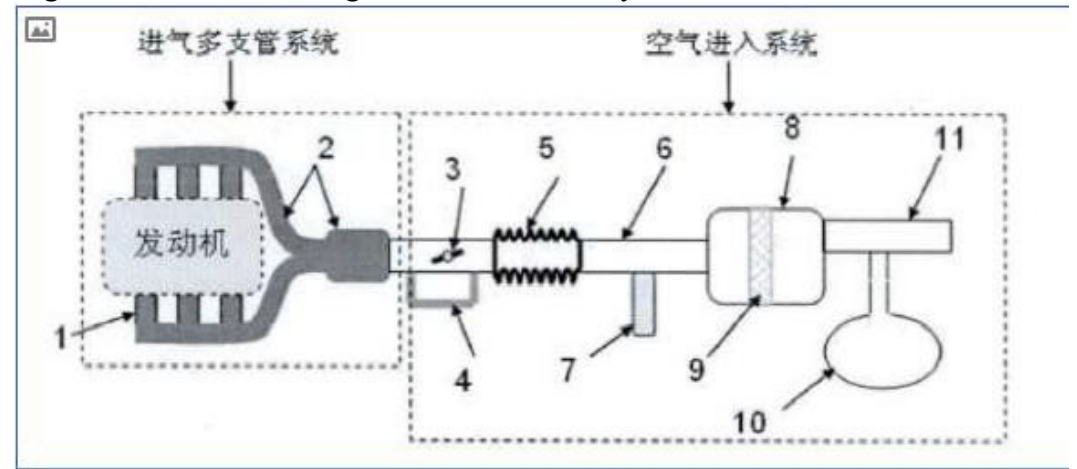

Note: 1-intake system, 2-intake manifold, 3-intake control valve, 4-idle intake channel, 5-flexible connecting pipe,6-clean air valve, 7-1/4 wavelength tube, 8 -air filter, 9-air filter, 10-Hermes muffler, 11-air inlet

Air through the air filter impurities, the flow through the air flow meter, through the inlet into the intake manifold, and the fuel injection of gasoline injected into the appropriate proportion of combustible mixture, by the intake valve into Gas within the ignition combustion, generating power. Thus, the engine power, torque and other dynamic performance and effective fuel consumption rate and other economic performance and the merits of the intake system is directly related.

To study the engine's intake system, a very important indicator is its acoustic performance. As mentioned earlier, the engine noise source, the intake system produces a large proportion of noise. To reduce the engine intake system noise, also need to be balanced the conflict between the engine power and acoustic performance.

The intake system is connected to the air compressor of the engine. The air pressure at the intake valve forms a pressure difference with the atmospheric pressure. The higher the pressure difference, the more energy the air will consume when entering the intake system. The power of the engine is related to the cross-sectional area of the intake pipe. The smaller the cross-sectional area is, the larger the power loss and the air flow will become smoothless. but the noise of the intake port is lower. Therefore, reducing the intake port noise at the same time may cause the engine power loss increases in the intake system design needs to balance this contradiction.

\subsubsection{Air intake noise generation mechanism}

After the air enters the inlet, its pressure and density will fluctuate as the engine intake valve closes periodically to form aerodynamic noise. According to their different mechanism, the noises are in four categories: the performance of the noise, the cyclical pressure pulsation noise, the gas rainbow Helmholtz resonance noise and the air column resonance noise. (1) bad noise

Air flow through the intake airway to achieve a high speed, due to the gas passage inside the valve and the inner wall of the trachea, burr and other obstacles exist, the flow of obstacles to the formation of calamity, resulting in noise. As the frequency of the spill will affect the frequency of such noise, the shape of the obstacle will affect the arrangement of the wicked, so the peak of its spectrum distribution in a specific width of the frequency range. However, when the obstruction of the flow around the regular geometry, the rotation of the formation, fall off and arranged in the flow stability of a certain periodicity, then the peak can be calculated according to the formula:

$$
f=\frac{s h \cdot v}{d} i(H z)
$$

Among them, the sh-constant, usually take 0.5 , v-intake air velocity $(\mathrm{m} / \mathrm{s})$, d-intake diameter $(\mathrm{m})$ 
(2) periodic pressure pulsation noise

Fresh air from the inlet into the intake pipe, the engine in the process of opening and closing the valve caused by air pressure and speed fluctuations, the formation of periodic changes in density, results in periodic pressure pulsation noise. It constitutes the main part of the intake noise, and the inlet pressure pulse coincidence, the main frequency $f$ by the engine speed, the formula is:

$$
f=\frac{2 n i}{60 \tau}(H z)
$$

Among them, n-engine speed (rpm), $\tau$-stroke number, i-engine cylinder number.

(3) Helmholtz resonance noise

In the engine work process, the gas will form a closed cavity, Helmholtz resonance noise on the resulting. The pressure wave of the gas in the gas source will stimulate the resonance, volume, inlet diameter and length of the noise to affect the size of the resonant frequency. The resonant cavity is equivalent to an equal section tube and a sphere with a certain volume. The first-order natural frequency is:

$$
f=\frac{c}{2 \pi} \sqrt{\frac{\pi r^{2}}{V_{h}\left(1+\frac{r}{2}\right)}}
$$

Among them, c-air velocity of sound (m/s), r-intake pipe radius, l-intake pipe length, - gas rainbow volume.

(4) Air column resonance noise in the intake pipe

After the intake valve is closed, the air intake pipe forms a gas column resonance system, and the air in the pipe is

$f=\frac{(2 i-1) c}{4 l}$

Where:

c was the air velocity of sound $(\mathrm{m} / \mathrm{s})$,

i was the harmonic number,

1 was the intake pipe length $(\mathrm{m})$.

\subsection{Optimization of intake muffler components}

In order to get a better-silencing effect, the following improvements to the silencer model:

(1) the appropriate increase in the length of the air filter, in the GT-Power will be between the outlet to the throttle between the use of straight pipe and cross-section pipe to replace the original three-dimensional cross-section tube.

(2) Since the change of the intake air temperature during the operation of the engine will cause the change of the flow rate, the size of the Helmholtz resonator and 1/4 wavelength tube will be adjusted according to the change of the flow rate during modeling. Optimized air intake system muffler components and engine said, simulation results are shown in Figure 2 and Figure 3.

It can be seen from Figure 2-2 and Figure 2-3 that the total sound pressure level of the inlet noise is below the target value, and the sound pressure level of each stage is lower than the target value, which is lower than that of the preoptimized inlet Gas pressure to the throttle pressure loss of $2.5 \mathrm{kPa}$, the optimized after the inlet noise and pressure loss all meet the requirements, the optimization effect is good. 


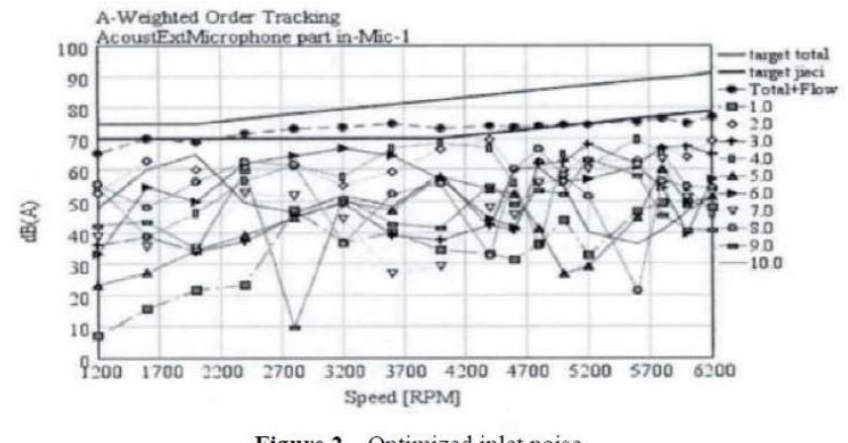

Figure 2. Optimized inlet noise

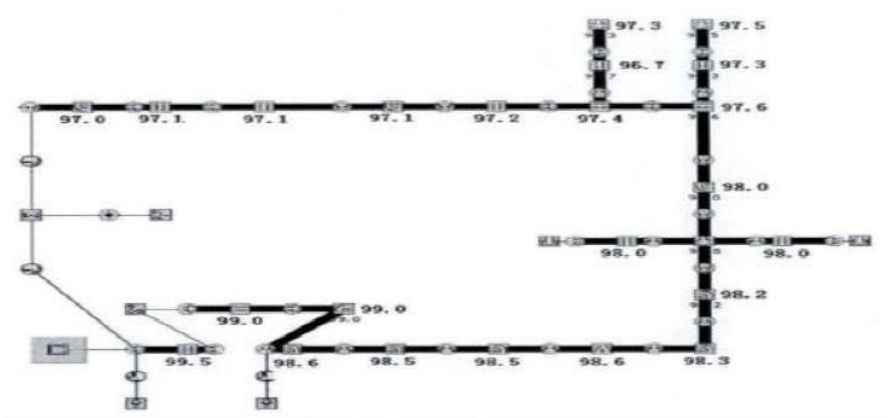

Figure 3. Optimized pressure loss of the intake system

\section{B15 Engine Air Intake System Performance Analysis}

\subsection{Establishment of flow analysis model for intake manifold}

Under the premise of satisfying the performance of the engine, the research on the noise reduction effect of the intake system silencer is not obvious for the other part of the intake system - the intake manifold because of its noise reduction effect. Field analysis discusses the role of the intake manifold structure in the engine intake process.

\subsubsection{Basic theory of computational fluid dynamics}

The STAR-CCM + software used in this chapter is the CFD software from the CD-adapco Group, which uses state-ofthe-art continuous medium mechanics technology to import meshes generated using the current mainstream mesh generation software, which itself can also calculate polyhedra Grid [5]. It combines with the widely used software engineering technology, has excellent performance and high reliability is a powerful tool for hot fluid analysis. At present, the software has become a lot of companies to flow field analysis and other aspects of the first choice of computational fluid mechanics of the basic idea is: with a limited number of discrete points on the variable value instead of the velocity field, force field and other continuous physical field, Variable value of the system of equations, and to solve the approximate value. In the calculation of hydrodynamics, we must observe three basic laws of conservation of the law, namely the law of conservation of momentum, the law of conservation of energy and the law of conservation of mass. In addition, if there are different components involved, the system must also adhere to the law of conservation of components; if there is turbulence, but also attached to the flow of the flow equation. In these conservation equations, the mass conservation equation is the law that all flow problems need to be satisfied. The formula is expressed as:

$$
\frac{\partial \rho}{\partial t}+\frac{\partial(\rho v)}{\partial \chi}+\frac{\partial(\rho v)}{\partial y}+\frac{\partial(\rho v)}{\partial z}=0
$$

$\mathrm{Or}$

$$
\frac{\partial \rho}{\partial t}+\nabla \cdot(\rho u)=0
$$

The momentum conservation equation is expressed as follows:

$$
\frac{\partial \rho U}{\partial t}+\nabla \cdot(\rho U \otimes U)=\nabla \cdot\left(-\rho \delta+\mu\left(\nabla U+(\nabla U)^{T}\right)\right)+S_{M}
$$

The energy conservation equation is expressed as follows:

$$
\frac{\partial \rho h_{\boldsymbol{w}}}{\partial t}-\frac{\partial p}{\partial t}+\nabla \cdot(\rho b \boldsymbol{w})=\nabla \cdot(\lambda \nabla T)+S_{E}
$$




\subsubsection{Inlet manifold flow field analysis method}

In the common flow field analysis and calculation, the zero-dimensional or one-dimensional model is usually used to simplify the intake manifold. For example, in the GT-Power software, the branch pipes of the intake manifold are simplified into several sections, One-dimensional connection to establish the model, its performance analysis. Each manifold of the intake manifold has a more obvious one-dimensional flow characteristic, with the software in the onedimensional module instead, the average pressure inside the regulator chamber is uniform. The calculated results of this method are less accurate than the experimental data, and cannot fully reflect the working condition of the manifold when the engine is running. Especially the flow field distribution in the manifold is difficult to give the exact conclusion. This is because the method does not consider the difference between the internal physical structures, suitable for low air flow rate, the pressure fluctuations of small occasions. In order to obtain one-dimensional software is difficult to achieve the intake manifold flow velocity and pressure distribution, CFD software is now widely used for simulation analysis, such as the aforementioned STAR-CCM + software. The model of the intake manifold is drawn and simplified by 3D modeling software. The CFD software is used to extract the fluid domain and divide the grid. After importing the grid, make the necessary settings to complete the calculation by adding the boundary conditions. CFD software can be used for steadystate flow field calculation, this method is less work, the calculation time is relatively short, can quickly obtain the flow characteristics within the manifold. However, this method cannot calculate the manifold at different times in the flow field, so when considering the role of time, you can select the transient calculation method. When using STAR-CCM + for transient calculations, it is necessary to obtain the boundary condition from the one-dimensional software or the test data.

After the model is set, the transient flow inside the manifold is obtained, which is closer to the real flow than the steady state because the boundary conditions are manually entered by the software user, cannot be based on the working conditions of the engine real-time updates, which is isolated from the transient results. The actual working process of the engine is transient, only CFD software cannot be used to simulate the actual state of the actual flow field. As the intake manifold and other parts of the thermodynamic system were separately solved, the two parts of the information cannot be exchanged in real time, compared with the actual test data, the accuracy of the whole system has not been greatly improved. Taking into account the GT-Power software can be more accurate simulation of the engine work process, in the flow manifold flow field analysis, the literature proposed another method of research - called the calculation. First use the GTPower software to build the engine model and calibration, so that the accuracy to meet the requirements. The intake manifold part of the model is replaced with a 3D module, and the corresponding interface is established in the STAR-CCM + software to obtain the real-time condition of the engine working process as its calculated boundary. This one-dimensional and three-dimensional software paste calculation method can make up for the use of three-dimensional software alone to set the conditions cannot be updated real-time, so that the boundaries of STAR-CCM + software in the GT-Power process to change at any time, thus greatly improving the model Accuracy. This chapter is the use of pot method, analysis of B15 engine intake manifold flow field.

\subsection{Vehicle Acceleration Noise Analysis}

In order to evaluate the merits of the design of the muffler element better, the corresponding model is lished by using the method of vehicle acceleration noise outside the vehicle, and the calculation result is analyzed and summarized. It is outside the noise contribution rate.

\subsubsection{Theoretical introduction}

According to GB1495-2002 'vehicle acceleration outside the vehicle noise limits and measurement methods' in the provisions of the car noise is based on the car to accelerate through the measurement area shown in Figure 3-1, when the noise outside the car to evaluate. During the measurement process, the relative position of the car and the sound level meter is changing, the measurement result of the noise is affected, and the engine noise characteristic also changes during the acceleration process. In Figure 4, the $\mathrm{O}$ point is the center of the measurement area, the acceleration section length is $2 \mathrm{X}$ $(10 \pm 0.05) \mathrm{m}$, the AA 'line is the starting line, the BB' line is the acceleration terminal line, the vehicle travels along the 
driving center line, The sensor layout is $(1.2 \pm 0.02) \mathrm{m}$ above the ground and CC' $(7.5 \pm 0.05) \mathrm{m}$ from the travel centerline. The reference axis must be horizontal and perpendicular to the travel center line CC. When the front end of the car is required to reach the AA line, the throttle is fully closed and remains unchanged through the measuring area. When the vehicle accelerates, the maximum noise level outside the vehicle should not exceed the limits specified by the standard.

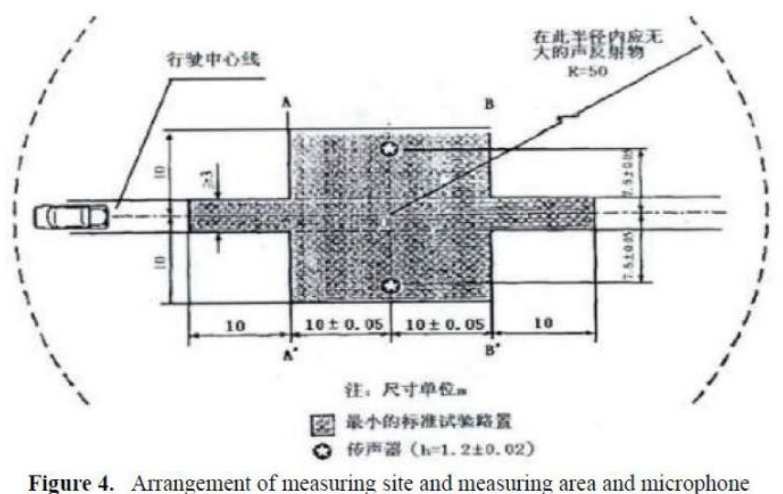

\subsubsection{Model establishment}

Through the noise model, use the 'Vehicle', 'Clutch Conn', 'Tire Conn Rigid', 'Acoust Microphone' and other modules, according to the test parameters to be set. Wherein the selection of the initial speed of the vehicle entry area is determined based on the vehicle matching the engine. For the setting of the microphone position, refer to the 'Vehicle Acceleration Noise Measurement Method and Measurement Method'. Based on the above settings, the vehicle accelerates the vehicle exterior noise model, as shown in Figure 5.

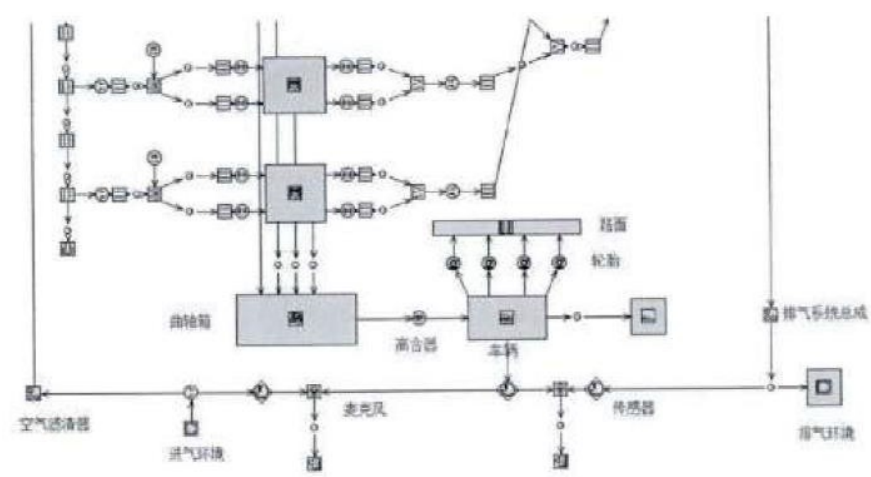

Figure 5. Vehicle Accelerated Vehicle Exterior Noise Model

\subsubsection{Analysis of results}

Figure 6 calculates the vehicle outside noise level calculated from the model in Figure 5. The X axis corresponds to the vehicle travel distance $(\mathrm{m})$ and the engine speed (rpm), respectively.

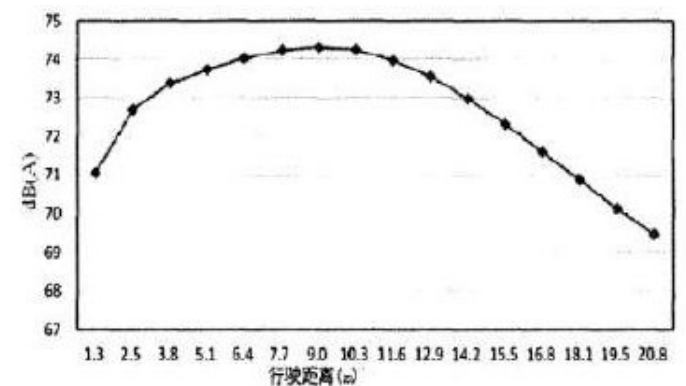

（a）随距离变化

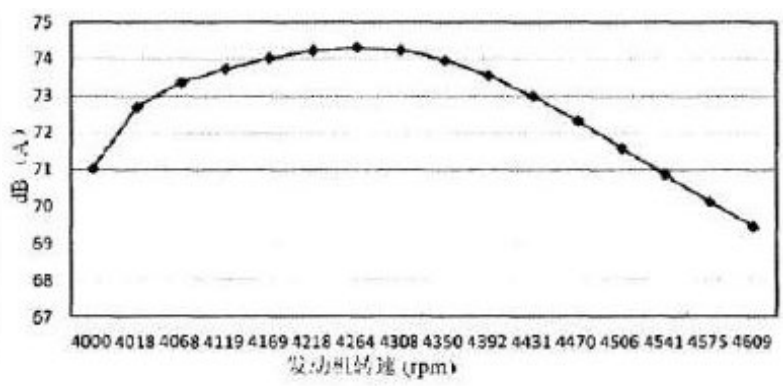

(b) 随动机转速变化

Figure 6. Vehicle acceleration noise outside the vehicle. 
From the experience of the test, we can see that when the vehicle accelerates driving through the measurement area, the maximum noise should be about $10 \mathrm{~m}$ away from the center of the accelerating section closest to the microphone. In this paper, the maximum noise appears in the vehicle travel distance of $9.0 \mathrm{~m}$ and $10.3 \mathrm{~m}$, about $74.3 \mathrm{~dB}$ (A). Since the sound pressure $(\mathrm{P})$ of the point source radiation is inversely proportional to the distance (1), the sound pressure SPL2 (dB (A)) of the more distant $\mathrm{r} 2$ can be calculated from the known sound pressure SPL1 (dB (A)) of the close r1 Out:

$$
S P L_{2}=S P L_{1}-D * \log \left(r_{2} / r_{1}\right)
$$

Where $\mathrm{r} 1-0.5$ is located at $500 \mathrm{~mm}$ at the air intake of the microphone device; $\mathrm{r} 2=7.5 \mathrm{~m}$, is located at $7.5 \mathrm{~m}$ at the microphone position. According to the formula available, the noise of the intake port can be converted by this formula. In addition, by referring to the literature, the noise level limit for the vehicle intake system is $63 \mathrm{~dB}$ (A) for the target noise of $75 \mathrm{~dB}(\mathrm{~A})$.

At present, China's noise regulations in January 1, 2005 after the production of Mi class car through the noise does not exceed $74 \mathrm{~dB}(\mathrm{~A})$. The maximum noise figure is $85.3 \mathrm{~dB}(\mathrm{~A})(4000 \mathrm{rpm}, 500 \mathrm{~mm})$ and the noise at $7.5 \mathrm{~m}$ is $100.3-23.5=$ 61.8 at the air inlet $500 \mathrm{~m}$ at the operating speed range of the engine. DB (A), less than 62dB (A) (74- (75-63)). Thus, the intake air noise of this engine satisfies the target value of the noise through the individual contribution of the noise.

\section{Summary and Prospect}

\subsection{Summary of the full text}

The application of computer-supported numerical simulation technology can greatly shorten the product development process and save the cost. It is widely used in various industries. In the automotive industry, due to national regulations on the requirements of automotive noise more stringent, only by improving the engine exhaust noise cannot meet the requirements, so the intake system noise is imperative. The use of numerical simulation technology to study the acoustic performance of the engine intake system and the design of the corresponding products, the specific design of some of the structure optimization is the current intake and exhaust system research more concerned about a topic.

The main task of this paper is to establish an accurate engine model. By analyzing the influence of the parameters of the muffler component on the transmission loss and the center frequency, the parameter sensitivity law is summed up, and the silencer components of the B15 engine are designed and optimized. And the one-dimensional and three-dimensional software coupling method is used to complete the analysis of the flow field of the intake manifold. Finally, a more accurate vehicle exterior noise model is established, and the contribution rate of the intake noise to the acceleration of the driving outside noise is analyzed. It is Muffler performance of intake muffler.

Summed up the full text of the work, mainly in the following areas:

(1) The use of GT-Power software to build the engine simulation process, the variable valve and variable intake manifold modeling in detail. The calculation results are compared with the experimental results of the external characteristics of the engine, and the model is calibrated to make the calculated value and the experimental value less than $5 \%$, which meet the accuracy requirement and lay the model foundation for the following design and analysis of the intake system.

(2) Using GT-Power to analyze the parameter sensitivity of the expansion muffler and the bypass muffler, the influence of the different structural parameters of the muffler on the muffler performance is summarized. Design the air filter, the Helmholtz resonator and the $1 / 4$ wavelength tube for the design requirements of the B15 engine intake system silencer components. The muffler element is coupled with the engine model to calculate the inlet system pressure loss and inlet noise, respectively. For the problem of slightly higher order noise at a certain frequency, considering the effect of temperature on the actual flow rate, an improvement scheme is proposed to make the inlet noise lower than the target limit; the inlet pressure loss is not higher than $2.5 \mathrm{kPa}$. The program meets the design requirements. 
(3) Introduce the three methods of analyzing the flow field of the intake manifold and select the manifold analysis by onedimensional and three-dimensional software coupling. Coupled GT-Power software with STAR-CCM + software, obtained real-time boundary conditions by GT-Power software, and analyzed pressure field and velocity field results.

(4) On the basis of the coupled GT-Power model, we add the pavement and tire modules to establish the vehicle exterior noise evaluation model, and analyze the contribution of the intake noise to the acceleration of the vehicle's driving outside noise. It is Muffler performance of air muffler. The results show that the design of the intake system muffler element is effective and satisfies the target requirement. Through the research of this paper, it is found that the numerical simulation method is used to design the engine parts, which is more targeted, which greatly saves the development cost, shortens the development cycle and provides guidance for the subsequent design and development.

\subsection{Outlook}

The use of numerical simulation method to simulate the engine work process and the development and design of parts, modern automobile industry development and promotion of new products, the necessary technical means. In this article, the design of the silencer components of the engine intake system and the performance analysis of the intake manifold are carried out by using the GT-Power and STAR-CCM + software. The proposed research methods and design schemes have achieved satisfactory results in the simulation Effect, with a certain practical value. However, from the current research situation, to achieve accurate design and high accuracy of the simulation, but also need more in-depth research and practice: (1) The use of GT-Power software for engine modeling, the relative position of the three-dimensional space is not reflected. In order to improve the simulation accuracy, you can use the GEM3D module to establish an accurate threedimensional model, its discrete into GT-Power for simulation. In addition, only consider the whole situation of the throttle valve cannot fully evaluate the performance of the engine, for part of the load when the simulation of the work process to be studied.

(2) Using STAR-CCM + software to calculate the flow field of the intake system, it is possible to analyze the entire intake system to which the intake muffler is added, rather than being limited to the analysis of the intake manifold. In addition, the structure of the individual manifolds in the manifold can be properly optimized and explored in depth.

\section{References}

1. Rayleigh J.W.S. The Theory of Sound. Volum n [M]. New York: Dover Publications, 1945

2. Shao Enpo, Cheng Hanhua. Mechanism and control of engine intake air noise [J], small internal combustion engine, 1994

3. Liu Sheng. Vehicle Gasoline Engine Inlet and Exhaust System Noise Simulation [D], Hefei University of Technology, 2007

4. Bhattacharya. Design of reactive mu ffl er for study on the noise level and performance of a two cylinder four stroke 16 h.p diesel engine, Noise and Vibration Worldwide, 2008

5. Bai Jie. Engine manifold catalytic converter heat fl ow coupling analysis [M], Wuhan University of Technology, 2011

6. Zhan Zhangsong, Chen Xiaodong, Zhang Xiaoyan. Application of GT-Power to Optimize Engine Inlet and Exhaust System Noise [J]. Changan Automobile Engineering Research Institute 